\title{
Getting Tie(2)d up in angiogenesis
}

\author{
Markus Ramsauer and Patricia A. D'Amore \\ Schepens Eye Research Institute, and Harvard Medical School, Boston, Massachusetts, USA \\ J. Clin. Invest. 110:1615-1617. (2002). doi:10.1172/JCI200217326.
}

Angiogenesis, the growth of new vessels from pre-existing blood vessels, requires complex signaling pathways and a high degree of spatial and temporal coordination among various cell types, multiple pro- and anti-angiogenic factors, and their corresponding receptors. Although the targeted disruption of many of these factors leads to embryonic lethal defects in vascular development, the precise actions of these factors at the cellular and molecular levels are not completely defined. Such is the case for angiopoietin-1 (Ang1), angiopoietin-2 (Ang2) and the Ang receptor Tie2. Mice deficient in Ang1, Ang2, or Tie2 exhibit aberrant vascular development, characterized by abnormal interactions between endothelial cells (ECs) and their supporting cells. The abluminal cells of the vasculature, which includes pericytes in the microvasculature and smooth muscle cells (SMCs) in large vessels, are collectively referred to as mural cells (reviewed in ref. 1). In this issue of the JCI, Uemura and colleagues provide some insight into the mechanisms that underlie these defects (2). The authors demonstrate that whereas blocking the function of the PDGF $\beta$ receptor (PDGFR- $\beta$ ) in the developing retinal vasculature led to mural cell-deficient vessels that

Address correspondence to: Patricia A. D’Amore, Schepens Eye Research Institute, 20 Staniford Street, Boston, Massachusetts 02114, USA. Phone: (617) 912-2559; Fax: (617) 912-0128;

E-mail: pdamore@vision.eri.harvard.edu.

Conflict of interest: The authors have

declared that no conflict of interest exists.

Nonstandard abbreviations used:

angiopoietin (Ang); endothelial cell (EC); smooth muscle cell (SMC); PDGF $\beta$ receptor (PDGFR- $\beta$ ); recombinant modified angiopoietin-1 (Ang1*); VEGF receptor 2 (VEGFR2). were poorly remodeled and leaky, administration of recombinant modified angiopoietin-1 (Ang1*) restored the vascular structure of the larger vessels in the absence of the mural cells.

\section{Vessel assembly}

The relevance of this observation is best appreciated in the context of the complexity of vessel assembly. During vascularization, a capillary plexus formed by vasculogenesis is remodeled via proliferation and migration of ECs (Figure 1). VEGF is essential in both developmental and postnatal (normal and pathologic) angiogenesis (reviewed in ref. 3). During murine retinal vascularization, the developmental process used by Uemura and coworkers, growth of the vessel sprouts is directed by astrocyte production of VEGF (4). It is important

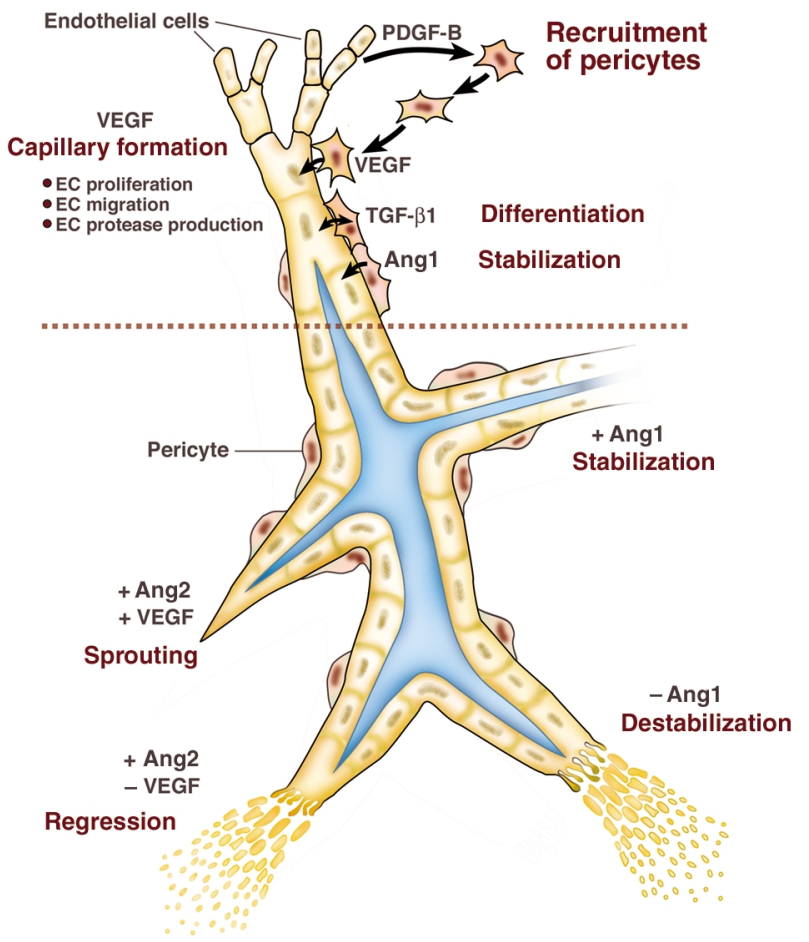

Figure 1

The multifactorial regulation of vessel assembly. The upper panel summarizes observations obtained using tissue culture models and knockout mice. Formation of a primary capillary network is initiated by VEGF-induced proliferation and migration of endothelial cells (ECs). Endothelial secretion of PDGF-B attracts mesenchymal cells, which contact the EC. Contact between the ECs and mesenchymal cells activates TGF- $\beta 1$, which suppresses endothelial proliferation and migration, induces mural cell differentiation, and is associated with vessel maturation. The lower panel illustrates findings in experimental models in which levels of angiopoietins were manipulated by exogenous administration. Addition of Ang1 results in stabilization of vessels in the absence of pericytes, whereas addition of Ang2 induces vessel regression in the absence of VEGF (-) and sprouting in the presence of VEGF $(+)$. 
to point out that the site of VEGF production varies among tissues and reflects tissue architecture (discussed in ref. 5). The nascent vessel is then modeled from its immature, unstable state to a mature, stable state. Proliferating ECs secrete PDGF-B, which acts as a chemoattractant and mitogen for undifferentiated mural cell precursors $(6,7)$. Mice deficient in PDGF-B or the PDGFR- $\beta$ display aberrant vessel remodeling and an absence of pericytes in the microvasculature (8). Tissue culture studies have demonstrated that contact between the ECs and newly-arrived mural cell precursors leads to the activation of TGF- $\beta 1$, which inhibits EC proliferation and migration $(9,10)$, reduces VEGF receptor 2 (VEGFR2) expression on ECs (11), and induces the differentiation of perivascular cells to a pericyte/SMC fate (6). In support of a key role for TGF- $\beta 1$, the targeted disruption of TGF- $\beta 1$, TGF$\beta R 2$ or the co-receptor endoglin, leads to defects in vascular development, apparently due to defects in epithelialmesenchymal interactions (12-14).

Recent studies have shown that concomitant with their differentiation, pericytes are induced to express VEGF, which may contribute to vessel stability by replacing VEGF supplied by the developing tissue itself (Darland and D'Amore, unpublished observations). The concept of VEGF dependence of nascent vessels is supported by several observations. Inhibition of endogenous VEGF in the pupillary membrane, a transient ocular microvessel network, via introduction of soluble VEGFR2, resulted in a significant increase in the number of apoptotic ECs (15). In addition, examination of new vessels induced in adult heart or liver by tissue-specific overexpression of VEGF revealed "a critical transition point beyond which remodeled new vessels persisted for months" in the absence of exogenous VEGF, whereas earlier withdrawal of VEGF led to vessel regression (16). Although not directly assessed in this study, the authors speculated that pericyte/SMC association with vessels would be associated with the observed functional stability. Interestingly, we have observed that pericytes associated with newly formed retinal vessels express VEGF, perhaps providing one mechanism by which the vessel stabil- ity is accomplished (Darland and D'Amore, unpublished observations).

\section{The role of the angiopoietins in vessel remodeling}

Somewhere in the remodeling process lies the contribution of the angiopoietins. A clue to the role of the angiopoietins is provided by examination of the expression patterns of the angiopoietins and their receptor Tie2 (reviewed in ref. 1). All ECs express Tie2 during development and in adult tissues, and Tie2 expression is increased during adult vascularization, such as during follicular development. Ang1 is expressed by mesenchyme and SMCs of the developing vasculature and continues to be expressed in adult organs that undergo vascular remodeling, such as the ovary. Ang2 expression in the adult, on the other hand, is restricted to sites of vascular remodeling. This pattern of expression led to a hypothesis that Ang1 is involved in vessel stabilization whereas Ang2 is a destabilizing factor.

More recently, this concept has evolved to consider the context in which the angiopoietins function in vivo. For example, in a tumor model (17) as well as in the pupillary membrane (18), Ang2 in the presence of endogenous VEGF promotes a rapid increase in capillary diameter, remodeling of the basal lamina, and new vessel growth. In contrast, if the activity of VEGF is blocked, Ang2 leads to EC death and vessel regression. Use of a 3-D spheroidal coculture as an in vitro model of vessel maturation demonstrated that direct contact between ECs and mesenchymal cells abrogates responsiveness to VEGF, whereas the presence of VEGF and Ang2 induced sprouting (19). These observations support a model in which the presence or absence of VEGF alters the action of Ang2 from anti-angiogenic to pro-angiogenic (Figure 1, lower panel).

The report of Uemura and colleagues now adds to these observations by demonstrating that addition of Ang ${ }^{*}$ to the developing retinal vasculature induces a degree of vessel maturation that can partially replace the effect of the associated mural cell. It should be noted that the Ang1* used in these studies is a variant of native Ang1 and is reportedly not sus- ceptible to inhibition by Ang2 (20), and thus may have effects that differ from the native molecule. Although the work of Uemura et al. do provide convincing evidence that at least a portion of the stabilizing action of the pericyte is mediated by Ang1, the precise means by which this cell effect is achieved remains unknown. Furthermore, as the authors themselves point out the "rescue affected by the addition of Ang $1^{*}$ of the pericyte-free retinal vasculature was incomplete, indicating involvement of other mural cell-derived molecules in the fine-tuning of vascular networks." (2). Thus, the association of the pericyte with the vessel accomplishes more than local Ang1 delivery. The activation of TGF- $\beta 1$ and its pluripotent actions as well as various junctions that form between ECs and pericytes (via cadherins and gap junctions) are likely to mediate additional heterotypic signaling between the endothelium and mural cells. A clear understanding of the role of pericytes in the maintenance of normal vessel stability is important for anti-angiogenic therapies aimed at vessel regression.

1. Loughna, S., and Sato, T.N. 2001. Angiopoietin and Tie signaling pathways in vascular development. Matrix Biol. 20:319-325.

2. Uemura, A., et al. 2002. Recombinant angiopoietin-1 restores higher-order architecture of growing blood vessels in mice in the absence of mural cells. J. Clin. Invest. 110:1619-1628. doi:10.1172/JCI200215621.

3. Ferrara, N. 2001. Role of vascular endothelial growth factor in regulation of physiologica angiogenesis. Am. J. Physiol. Cell Physiol. 280:C1358-C1366.

4. Stone, J., et al. 1995. Development of retinal vasculature is mediated by hypoxia-induced vascular endothelial growth factor (VEGF) expression by neuroglia. J. Neurosci. 15:4738-4747.

5. D'Amore, P.A., and Ng, Y.S. 2002. Won't you be my neighbor? Local induction of arteriogenesis. Cell. 110:289-292.

6. Hirschi, K., Rohovsky, S.A., and D'Amore, P.A 1998. PDGF, TGF- $\beta$ and heterotypic cell-cell interactions mediate the recruitment and differentiation of $10 \mathrm{~T} 1 / 2$ cells to a smooth muscle cell fate. J. Cell. Biol. 141:805-814.

7. Hirschi, K.K., Rohovsky, S.A., Beck, L.H., Smith, S.R., and D'Amore, P.A. 1999. Endothelial cells modulate the proliferation of mural cell precursors via platelet-derived growth factor-BB and heterotypic cell contact. Circ. Res. 84:298-230.

8. Hellstrom, M., Kalen, M., Lindahl, P., Abramsson, A., and Betsholtz, C. 1999. Role of PDGF-B and PDGFR- $\beta$ in recruitment of vascular smooth muscle cells and pericytes during embryonic blood vessel formation in the mouse. Development. 126:3047-3055.

9. Orlidge, A., and D'Amore, P.A. 1987. Inhibition of capillary endothelial cell growth by pericytes and smooth muscle cells. J. Cell Biol. 105: $1455-1462$.

10. Sato, Y., and Rifkin, D.B. 1989. Inhibition of endothelial cell movement by pericytes and 
smooth muscle cells: activation of a latent transforming growth factor-beta 1-like molecule by plasmin during co-culture. J. Cell Biol. 109:309-315.

11. Mandriota, S., Menoud, P.-A., and Pepper, M. 1996. Transforming growth factor b1 down-regulates vascular endothelial growth factor $2 / f l k-1$ expression in vascular endothelial cells. J. Biol. Chem. 271:11500-11505.

12. Oshima, M., Oshima, H., and Taketo, M.M. 1996. TGF- $\beta$ receptor type II deficiency results in defects of yolk sac hematopoiesis and vasculogenesis. Dev. Biol. 179:297-302.

13. Dickson, M.C., et al. 1995. Defective haematopoiesis and vasculogenesis in transforming growth factor- $\beta 1$ knock-out mice. Development 121:1845-1854.

14. Li, D.Y., et al. 1999. Defective angiogenesis in mice lacking endoglin. Science. 284:1534-1537.

15. Meeson, A.P., Argilla, M., Ko, K., Witte, L., and Lang, R.A. 1999. VEGF deprivation-induced apoptosis is a component of programmed capillary regression. Development. 126:1407-1415.

16. Dor, Y., et al. 2002. Conditional switching of VEGF provides new insights into adult neovascularization and pro-angiogenic therapy. $E M B O$ J. 21:1939-1947.

17. Holash, J., et al. 1999. Vessel cooption, regression, and growth in tumors mediated by angiopoietins and VEGF. Science. 284:1994-1998.
18. Lobov, I.B., Brooks, P.C., and Lang, R.A. 2002. Angiopoietin-2 displays the VEGF-dependent modulation of capillary structure and endothelial survival in vivo. Proc. Natl. Acad. Sci. USA. 99:11205-11210.

19. Korff, T., Kimmina, S., Martiny-Baron, G., and Augustin, H.G. 2001. Blood vessel maturation in a 3-dimensional spheroidal coculture model: direct contact with smooth muscle cells regulates endothelial cell quiescence and abrogates VEGF responsiveness. FASEB J. 15:447-457.

20. Koblizek, T.I., Weiss, C., Yancopoulos, G.D. Deutsch, U., and Risau, W. 1998. Angiopoietin1 induces sprouting angiogenesis in vitro. Curr. Biol. 8:529-532. 